\title{
REGULAR DECAY OF BALL DIAMETERS AND SPECTRA OF RUELLE OPERATORS FOR CONTACT ANOSOV FLOWS
}

\author{
LUCHEZAR STOYANOV
}

(Communicated by Yingfei Yi)

\begin{abstract}
For Anosov flows on compact Riemann manifolds we study the rate of decay along the flow of diameters of balls $B^{s}(x, \epsilon)$ on local stable manifolds at Lyapunov regular points $x$. We prove that this decay rate is similar for all sufficiently small values of $\epsilon>0$. From this and the main result in an earlier paper, we derive strong spectral estimates for Ruelle transfer operators for contact Anosov flows with Lipschitz local stable holonomy maps. These apply in particular to geodesic flows on compact locally symmetric manifolds of strictly negative curvature. As is now well known, such spectral estimates have deep implications in some related areas, e.g. in studying analytic properties of Ruelle zeta functions and partial differential operators, asymptotics of closed orbit counting functions, etc.
\end{abstract}

\section{INTRODUCTION}

Consider a non-linear system of differential equations of the form

$$
\dot{x}(t)=f(t, x),
$$

where $f: U \times \mathbb{R} \longrightarrow \mathbb{R}^{n}$ is a continuously differentiable map for some open ball $U$ with center 0 in $\mathbb{R}^{n}$ and $f(t, 0)=0$ for all $t \in \mathbb{R}$. Assuming that the null solution of (1.1) is asymptotically stable (see e.g. [CL]), one defines a semi-flow $\varphi_{t}: U \times[0, \infty) \longrightarrow U$ such that $\varphi_{t}(z, s)=x(t+s)$, where $x$ is the solution of $(1.1)$ with $x(s)=z$. One may then ask the question whether for all sufficiently small $0<\delta_{1}<\delta_{2}$ there exists a constant $C>0$ depending only on $\delta_{1}$ and $\delta_{2}$ (and $f$ ) such that $\operatorname{diam}\left(\varphi_{t}\left(B\left(0, \delta_{2}\right)\right)\right) \leq C \operatorname{diam}\left(\varphi_{t}\left(B\left(0, \delta_{1}\right)\right)\right)$ for all $t \geq 0$, where $B(0, \delta)$ denotes the (closed) ball with center 0 and radius $\delta$ in $\mathbb{R}^{n}$. We do not know what happens in the general case; however, it follows from the arguments in the present paper that under a certain (Lyapunov regularity) condition at 0 , the answer to the above question is affirmative.

In fact, we consider a more complicated situation. Let $\phi_{t}: M \longrightarrow M$ be a $C^{2}$ Anosov flow on a $C^{2}$ compact Riemann manifold $M$. For any $x \in M$ and a sufficiently small $\delta>0$ consider the closed $\delta$-ball $B^{s}(x, \delta)=\left\{y \in W_{\epsilon}^{s}(x)\right.$ : $d(x, y) \leq \delta\}$ on the local stable manifold $W_{\epsilon}^{s}(x)$. For any $y \in W_{\epsilon}^{s}(x)$ we know that $d\left(\phi_{t}(x), \phi_{t}(y)\right) \rightarrow 0$ exponentially fast as $t \rightarrow \infty$. Moreover, we have uniform estimates for the exponential rate of convergence, so for any $x \in M$ and any given $\delta>0, \operatorname{diam}\left(\phi_{t}\left(B^{s}(x, \delta)\right)\right) \rightarrow 0$ exponentially fast as $t \rightarrow \infty$. However, in general, it is not clear whether for any constants $0<\delta_{1}<\delta_{2}$ the ratio $\frac{\operatorname{diam}\left(\phi_{t}\left(B^{s}\left(x, \delta_{2}\right)\right)\right)}{\operatorname{diam}\left(\phi_{t}\left(B^{s}\left(x, \delta_{1}\right)\right)\right)}$ is

Received by the editors April 6, 2011.

2010 Mathematics Subject Classification. Primary 37D20, 37D25.

(C) 2012 American Mathematical Society 3463 
uniformly bounded for $t>0$ and $x \in M$ (although a similar property is obviously satisfied by the linearized flow $d \phi_{t}$, considering balls on corresponding tangent planes). It appears that in general this problem is rather subtle, and it is not clear at all whether one should expect a positive solution without any extra assumptions.

Here we consider a similar problem on the set $\mathcal{L}$ of Lyapunov regular points in $M$; see section 3.1 for the terminology. We prove the following.

Theorem 1.1. For every $\epsilon>0$ there exist Lyapunov $\epsilon$-regularity functions $\omega$ : $\mathcal{L} \longrightarrow(0,1)$ and $G: \mathcal{L} \longrightarrow[1, \infty)$ such that for any $0<\delta_{1}<\delta_{2}$ there exists a constant $K=K\left(\delta_{1}, \delta_{2}\right) \geq 1$ with $\operatorname{diam}\left(\phi_{t}\left(B^{s}\left(x, \delta_{2}\right)\right) \leq K G(x) \operatorname{diam}\left(\phi_{t}\left(B^{s}\left(x, \delta_{1}\right)\right)\right)\right.$ for all $x \in \mathcal{L}$ with $\delta_{2} \leq \omega(x)$ and all $t>0$.

A similar result can be proved for non-uniformly hyperbolic flows.

The above has an important consequence concerning cylinders in a symbolic coding of the flow defined by means of a Markov family; see Theorem 4.2 below for details.

The motivation for this work came from [St1, where assuming the properties (a) and (b) in Theorem 4.2 below, Lipschitzness of the local stable holonomy maps and a certain non-integrability condition, we prove strong spectral estimates for arbitrary potentials over basic sets for Axiom A flows, similar to those established by Dolgopyat $\mathrm{D}$ for geodesic flows on compact surfaces (for general potentials) and transitive Anosov flows on compact manifolds with $C^{1}$ jointly non-integrable horocycle foliations (for the Sinai-Bowen-Ruelle potential). It is known that such strong spectral estimates lead to deep results in a variety of areas which are difficult (if not impossible) to obtain by other means (see e.g. PoS1, PoS2, PoS3, An, [PeS1] PeS2], [PeS3]).

Let $\mathcal{R}=\left\{R_{i}\right\}_{i=1}^{k}$ be a Markov family for $\phi_{t}$ consisting of rectangles $R_{i}=\left[U_{i}, S_{i}\right]$, where $U_{i}$ (resp. $S_{i}$ ) are (admissible) subsets of $W_{\epsilon}^{u}\left(z_{i}\right)$ (resp. $W_{\epsilon}^{s}\left(z_{i}\right)$ ) for some $\epsilon>0$ and $z_{i} \in M$ (cf. section 2 for details). The first return time function $\tau: R=\bigcup_{i=1}^{k} R_{i} \longrightarrow[0, \infty)$ and the standard Poincaré map $\mathcal{P}: R \longrightarrow R$ are then naturally defined. Setting $U=\bigcup_{i=1}^{k} U_{i}$, the shift map $\sigma: U \longrightarrow U$ defined by $\sigma=\pi^{(U)} \circ \mathcal{P}$, where $\pi^{(U)}: R \longrightarrow U$ is the projection along the leaves of local stable manifolds, provides a natural symbolic coding of the flow. To avoid dealing with boundary points in $U$, consider the set $\widehat{U}$ of all $u \in U$ whose orbits do not have common points with the boundary of $R$ (see section 2 ).

Given a Lipschitz real-valued function $f$ on $\widehat{U}$, set $g=g_{f}=f-P \tau$, where $P=P_{f} \in \mathbb{R}$ is the unique number such that the topological pressure $\operatorname{Pr}_{\sigma}(g)$ of $g$ with respect to $\sigma$ is zero (cf. e.g. $[\mathrm{PP}$ ). For $a, b \in \mathbb{R}$, one defines the Ruelle transfer operator $L_{g-(a+\mathbf{i} b) \tau}: C^{\operatorname{Lip}}(\widehat{U}) \longrightarrow C^{\operatorname{Lip}}(\widehat{U})$ in the usual way (cf. section 2$)$. Here $C^{\operatorname{Lip}}(\widehat{U})$ is the space of Lipschitz functions $h: \widehat{U} \longrightarrow$ C. By $\operatorname{Lip}(h)$ we denote the Lipschitz constant of $h$ and by $\|h\|_{0}$ the standard $\sup$ norm of $h$ on $\widehat{U}$.

We will say that the Ruelle transfer operators related to the function $f$ on $U$ are eventually contracting if for every $\epsilon>0$ there exist constants $0<\rho<1, a_{0}>0$ and $C>0$ such that if $a, b \in \mathbb{R}$ satisfy $|a| \leq a_{0}$ and $|b| \geq 1 / a_{0}$, then for every integer $m>0$ and every $h \in C^{\operatorname{Lip}(\widehat{U})}$ we have $\left\|L_{f-\left(P_{f}+a+\mathbf{i} b\right) \tau}^{m} h\right\|_{\operatorname{Lip}, b} \leq C \rho^{m}|b|^{\epsilon}\|h\|_{\text {Lip }, b}$,

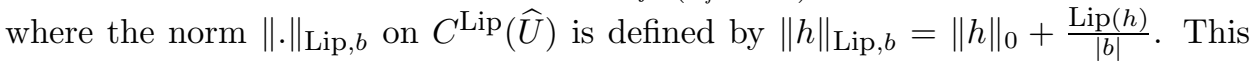
implies in particular that the spectral radius of $L_{f-\left(P_{f}+a+\mathbf{i} b\right) \tau}$ on $C^{\mathrm{Lip}}(\widehat{U})$ does not exceed $\rho$. 
From Theorem 1.1 (or rather its consequence, Theorem 4.2 below) and the main result in St1] we derive the following.

Theorem 1.2. Let $\phi_{t}: M \longrightarrow M$ be a $C^{2}$ transitive contact Anosov flow on a $C^{2}$ compact Riemann manifold with uniformly Lipschitz local stable holonomy maps. Then for any Lipschitz real-valued function $f$ on $U$ the Ruelle transfer operators related to $f$ are eventually contracting.

The reader is referred to section 2 below for the definition of local holonomy maps. In general these are only Hölder continuous. It is known that uniform Lipschitzness of the local stable holonomy maps can be derived from a certain bunching condition concerning the rates of expansion/contraction of the flow along local unstable/stable manifolds over $M$ (see [Ha], PSW]).

A result similar to Theorem 1.2 is true for general (not necessarily contact) Anosov flows; however, one has to assume in addition a local non-integrability condition (see condition (LNIC) in [St1]). Using a smoothing procedure as in [D], an estimate similar to that in Theorem 1.2 holds for the Ruelle operator acting on the space $\mathcal{F}_{\gamma}(U)$ of Hölder continuous functions with respect to an appropriate norm.

For geodesic flows on locally symmetric spaces of negative curvature it is well known that the local stable and unstable manifolds are smooth $\left(C^{\infty}\right)$, so the corresponding local holonomy maps are smooth as well. Thus, as an immediate consequence of Theorem 1.2 one obtains the following.

Theorem 1.3. Let $X$ be a compact locally symmetric space of negative curvature and let $\phi_{t}: M=S^{*}(X) \longrightarrow M$ be the geodesic flow on $X$. Then for any Lipschitz real-valued function $f$ on $U$ the Ruelle transfer operators related to $f$ are eventually contracting.

As mentioned above, there are various consequences that can be derived from results such as Theorem 1.2 (or Theorem 1.3). Here we state one of these.

As in St1, one can use Theorem 1.2 and an argument of Pollicott and Sharp PoS1] to get certain information about the Ruelle zeta function

$$
\zeta(s)=\prod_{\gamma}\left(1-e^{-s \ell(\gamma)}\right)^{-1},
$$

where $\gamma$ runs over the set of primitive closed orbits of $\phi_{t}$ and $\ell(\gamma)$ is the least period of $\gamma$. Let $h_{T}$ denote the topological entropy of $\phi_{t}$.

Corollary 1.4. Under the assumptions in Theorems 1.2 or 1.3 , the zeta function $\zeta(s)$ of the flow $\phi_{t}: M \longrightarrow M$ has an analytic and non-vanishing continuation in a half-plane $\operatorname{Re}(s)>c_{0}$ for some $c_{0}<h_{T}$ except for a simple pole at $s=h_{T}$. Moreover, there exists $c \in\left(0, h_{T}\right)$ such that

$$
\pi(\lambda)=\#\{\gamma: \ell(\gamma) \leq \lambda\}=\operatorname{li}\left(e^{h_{T} \lambda}\right)+O\left(e^{c \lambda}\right)
$$

as $\lambda \rightarrow \infty$, where $\operatorname{li}(x)=\int_{2}^{x} \frac{d u}{\log u} \sim \frac{x}{\log x}$ as $x \rightarrow \infty$.

In fact, a direct application of Theorem 5 in $[\mathrm{PeS} 3$ gives a more precise estimate of the number of closed trajectories of the flow with primitive periods lying in exponentially shrinking intervals. We refer the reader to section 6 in $\mathrm{PeS} 3$ ] for details. 
Section 2 contains some basic definitions and preliminary facts. In section 3 we compare diameters of balls with respect to Bowen's metric on unstable manifolds and prove the analogue of Theorem 1.1 for unstable manifolds. From this, Theorem 1.1 is derived easily. Finally in section 4 we consider cylinders in the set $U$ defined by means of a Markov family, and prove two properties of the decay rates of the diameters of such cylinders (Theorem 4.2), assuming that the local stable holonomy maps are uniformly Lipschitz. We do not know whether the same properties hold for any Anosov flow. Theorem 1.2 is then derived using Theorem 4.2 and the argument in section 5 of [St1].

\section{Preliminaries}

Throughout this paper $M$ denotes a $C^{2}$ compact Riemann manifold, and $\phi_{t}$ : $M \longrightarrow M(t \in \mathbb{R})$ a $C^{2}$ flow on $M$. The flow is called hyperbolic if $M$ contains no fixed points and there exist constants $C>0$ and $0<\lambda<1$ such that there exists a $d \phi_{t}$-invariant decomposition $T_{x} M=E^{0}(x) \oplus E^{u}(x) \oplus E^{s}(x)$ of $T_{x} M(x \in M)$ into a direct sum of non-zero linear subspaces, where $E^{0}(x)$ is the one-dimensional subspace determined by the direction of the flow at $x,\left\|d \phi_{t}(u)\right\| \leq C \lambda^{t}\|u\|$ for all $u \in E^{s}(x)$ and $t \geq 0$, and $\left\|d \phi_{t}(u)\right\| \leq C \lambda^{-t}\|u\|$ for all $u \in E^{u}(x)$ and $t \leq 0$. The flow $\phi_{t}$ is called an Anosov flow on $M$ if the periodic points are dense in $M$ (see e.g. $[\mathrm{KH}]$ ). The flow is called transitive if it has a dense orbit, and contact if there exists a $C^{2}$ flow invariant one-form $\omega$ on $M$ such that $\omega \wedge(d \omega)^{n}$ is nowhere zero, where $\operatorname{dim}(M)=2 n+1$.

For $x \in M$ and a sufficiently small $\epsilon>0$ let

$$
\begin{gathered}
W_{\epsilon}^{s}(x)=\left\{y \in M: d\left(\phi_{t}(x), \phi_{t}(y)\right) \leq \epsilon \text { for all } t \geq 0, d\left(\phi_{t}(x), \phi_{t}(y)\right) \rightarrow_{t \rightarrow \infty} 0\right\}, \\
W_{\epsilon}^{u}(x)=\left\{y \in M: d\left(\phi_{t}(x), \phi_{t}(y)\right) \leq \epsilon \text { for all } t \leq 0, d\left(\phi_{t}(x), \phi_{t}(y)\right) \rightarrow_{t \rightarrow-\infty} 0\right\}
\end{gathered}
$$

be the (strong) stable and unstable manifolds of size $\epsilon$. Then $E^{u}(x)=T_{x} W_{\epsilon}^{u}(x)$ and $E^{s}(x)=T_{x} W_{\epsilon}^{s}(x)$. Given $\delta>0$, set $E^{u}(x ; \delta)=\left\{u \in E^{u}(x):\|u\| \leq \delta\right\} ; E^{s}(x ; \delta)$ is defined similarly.

It follows from the hyperbolicity of the flow that if $\epsilon_{0}>0$ is sufficiently small, there exists $\epsilon_{1}>0$ such that if $x, y \in M$ and $d(x, y)<\epsilon_{1}$, then $W_{\epsilon_{0}}^{s}(x)$ and $\phi_{\left[-\epsilon_{0}, \epsilon_{0}\right]}\left(W_{\epsilon_{0}}^{u}(y)\right)$ intersect at exactly one point $[x, y] \in M$ (cf. $[\mathrm{KH}]$ ). That is, there exists a unique $t \in\left[-\epsilon_{0}, \epsilon_{0}\right]$ such that $\phi_{t}([x, y]) \in W_{\epsilon_{0}}^{u}(y)$. For $x, y \in M$ with $d(x, y)<\epsilon_{1}$, define $\pi_{y}(x)=[x, y]=W_{\epsilon}^{s}(x) \cap \phi_{\left[-\epsilon_{0}, \epsilon_{0}\right]}\left(W_{\epsilon_{0}}^{u}(y)\right)$. Thus, for a fixed $y \in M, \pi_{y}: W \longrightarrow \phi_{\left[-\epsilon_{0}, \epsilon_{0}\right]}\left(W_{\epsilon_{0}}^{u}(y)\right)$ is the projection along local stable manifolds defined on a small open neighbourhood $W$ of $y$ in $M$. Choosing $\epsilon_{1} \in$ $\left(0, \epsilon_{0}\right)$ sufficiently small, the restriction $\pi_{y}: \phi_{\left[-\epsilon_{1}, \epsilon_{1}\right]}\left(W_{\epsilon_{1}}^{u}(x)\right) \longrightarrow \phi_{\left[-\epsilon_{0}, \epsilon_{0}\right]}\left(W_{\epsilon_{0}}^{u}(y)\right)$ is called a local stable holonomy map 1

We will say that $A$ is an admissible subset of $W_{\epsilon}^{u}(z)(z \in M)$ if $A$ coincides with the closure of its interior in $W_{\epsilon}^{u}(z)$. Admissible subsets of $W_{\epsilon}^{s}(z)$ are defined similarly. As in [D], a subset $R$ of $\Lambda$ will be called a rectangle if it has the form $R=[U, S]=\{[x, y]: x \in U, y \in S\}$, where $U$ and $S$ are admissible subsets of $W_{\epsilon}^{u}(z)$ and $W_{\epsilon}^{s}(z)$, respectively, for some $z \in M$. In what follows we will denote by

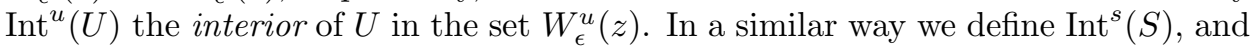
then set $\operatorname{Int}(R)=\left[\operatorname{Int}^{u}(U), \operatorname{Int}^{s}(S)\right]$. Given $\xi=[x, y] \in R$, set $W_{R}^{u}(\xi)=[U, y]=$ $\left\{\left[x^{\prime}, y\right]: x^{\prime} \in U\right\}$ and $W_{R}^{s}(\xi)=[x, S]=\left\{\left[x, y^{\prime}\right]: y^{\prime} \in S\right\} \subset W_{\epsilon_{0}}^{s}(x)$. The interiors

\footnotetext{
${ }^{1}$ In a similar way one can define holonomy maps between any two sufficiently close local transversals to stable laminations; see e.g. [PSW].
} 
of these sets in the corresponding leaves are defined by $\operatorname{Int}^{u}\left(W_{R}^{u}(\xi)\right)=\left[\operatorname{Int}^{u}(U), y\right]$ and $\operatorname{Int}^{s}\left(W_{R}^{s}(\xi)\right)=\left[x, \operatorname{Int}^{s}(S)\right]$.

Let $\mathcal{R}=\left\{R_{i}\right\}_{i=1}^{k}$ be a family of rectangles with $R_{i}=\left[U_{i}, S_{i}\right], U_{i} \subset W_{\epsilon}^{u}\left(z_{i}\right)$ and $S_{i} \subset W_{\epsilon}^{s}\left(z_{i}\right)$, respectively, for some $z_{i} \in M$. Since the set $\mathcal{L}$ of Lyapunov regular points (see section 3.1 below) is dense, without loss of generality we will assume that $z_{i} \in R_{i} \cap \mathcal{L}$. Set $R=\bigcup_{i=1}^{k} R_{i}$. The family $\mathcal{R}$ is called complete if there exists $T>0$ such that for every $x \in M, \phi_{t}(x) \in R$ for some $t \in(0, T]$. Given a complete family $\mathcal{R}$, the related Poincaré map $\mathcal{P}: R \longrightarrow R$ is defined by $\mathcal{P}(x)=\phi_{\tau(x)}(x) \in R$, where $\tau(x)>0$ is the smallest positive time with $\phi_{\tau(x)}(x) \in R$. The function $\tau$ is called the first return time associated with $\mathcal{R}$. A complete family $\mathcal{R}=\left\{R_{i}\right\}_{i=1}^{k}$ of rectangles in $M$ is called a Markov family of size $\chi>0$ for the flow $\phi_{t}$ if $\operatorname{diam}\left(R_{i}\right)<\chi$ for all $i$ and: (a) for any $i \neq j$ and any $x \in \operatorname{Int}\left(R_{i}\right) \cap \mathcal{P}^{-1}\left(\operatorname{Int}\left(R_{j}\right)\right)$ we have $\mathcal{P}\left(\operatorname{Int}^{s}\left(W_{R_{i}}^{s}(x)\right)\right) \subset \operatorname{Int}^{s}\left(W_{R_{j}}^{s}(\mathcal{P}(x))\right)$ and $\mathcal{P}\left(\operatorname{Int}^{u}\left(W_{R_{i}}^{u}(x)\right)\right) \supset \operatorname{Int}^{u}\left(W_{R_{j}}^{u}(\mathcal{P}(x))\right)$; (b) for any $i \neq j$ at least one of the sets $R_{i} \cap \phi_{[0, \chi]}\left(R_{j}\right)$ and $R_{j} \cap \phi_{[0, \chi]}\left(R_{i}\right)$ is empty.

The existence of a Markov family $\mathcal{R}$ of an arbitrarily small size $\chi>0$ for $\phi_{t}$ follows from the construction of Bowen $\mathrm{B}$. (cf. also Ratner $[\mathrm{Ra}]$ ).

From now on we will assume that $\mathcal{R}=\left\{R_{i}\right\}_{i=1}^{k}$ is a fixed Markov family for $\phi_{t}$ of small size $\chi<\epsilon_{0} / 2<1$. Set $U=\bigcup_{i=1}^{k} U_{i}$ and $\operatorname{Int}^{u}(U)=\bigcup_{j=1}^{k} \operatorname{Int}^{u}\left(U_{j}\right)$. The shift map $\sigma: U \longrightarrow U$ is given by $\sigma=\pi^{(U)} \circ \mathcal{P}$, where $\pi^{(U)}: R \longrightarrow U$ is the projection along stable leaves. Notice that $\tau$ is constant on each stable leaf $W_{R_{i}}^{s}(x)=W_{\epsilon_{0}}^{s}(x) \cap R_{i}$. For any integer $m \geq 1$ and any function $h: U \longrightarrow \mathbf{C}$ define $h_{m}: U \longrightarrow \mathbf{C}$ by $h_{m}(u)=h(u)+h(\sigma(u))+\ldots+h\left(\sigma^{m-1}(u)\right)$.

Denote by $\widehat{U}$ the core of $U$, i.e. the set of those $x \in U$ such that $\mathcal{P}^{m}(x) \in$ $\operatorname{Int}(R)=\bigcup_{i=1}^{k} \operatorname{Int}\left(R_{i}\right)$ for all $m \in \mathbb{Z}$. It is well known (see [B]) that $\widehat{U}$ is a residual subset of $U$ and has full measure with respect to any Gibbs measure on $U$. Clearly in general $\tau$ is not continuous on $U$; however, $\tau$ is essentially Lipschitz on $U$ in the sense that there exists a constant $L>0$ such that if $x, y \in U_{i} \cap \sigma^{-1}\left(U_{j}\right)$ for some $i, j$, then $|\tau(x)-\tau(y)| \leq L d(x, y)$. The same applies to $\sigma: U \longrightarrow U$.

Let $B(\widehat{U})$ be the space of bounded functions $g: \widehat{U} \longrightarrow \mathbf{C}$ with its standard norm $\|g\|_{0}=\sup _{x \in \widehat{U}}|g(x)|$. Given a function $g \in B(\widehat{U})$, the Ruelle transfer operator $L_{g}: B(\widehat{U}) \longrightarrow B(\widehat{U})$ is defined by $\left(L_{g} h\right)(u)=\sum_{\sigma(v)=u} e^{g(v)} h(v)$. If $g \in B(\widehat{U})$ is essentially Lipschitz on $\widehat{U}$, then $L_{g}$ preserves the space $C^{\operatorname{Lip}}(\widehat{U})$ of Lipschitz functions $h: \widehat{U} \longrightarrow \mathbf{C}$.

The hyperbolicity of the flow on $M$ and the additional assumption (in section 4 below) that the local stable holonomy maps are uniformly Lipschitz implies the existence of constants $c_{0} \in(0,1]$ and $\gamma_{1}>\gamma>1$ such that

$$
c_{0} \gamma^{m} d\left(u_{1}, u_{2}\right) \leq d\left(\sigma^{m}\left(u_{1}\right), \sigma^{m}\left(u_{2}\right)\right) \leq \frac{\gamma_{1}^{m}}{c_{0}} d\left(u_{1}, u_{2}\right)
$$

whenever $\sigma^{j}\left(u_{1}\right)$ and $\sigma^{j}\left(u_{2}\right)$ belong to the same $U_{i_{j}}$ for all $j=0,1, \ldots, m$.

\section{Comparison of Ball diameters}

3.1. Lyapunov regularity. Let $M$ be a $C^{2}$ compact Riemann manifold and $\phi_{t}$ an Anosov $C^{2}$ flow on $M$. Set $f=\phi_{1}$ and denote by $\mathcal{L}$ the set of all Lyapunov regular points of $f$ (see [P1] or section 2.1 in $[\mathrm{BP}]$ ). It is well known that $\mathcal{L}$ is dense in $M$ and has full measure with respect to any $f$-invariant probability measure on 
$M$. Let

$$
\lambda_{1}<\lambda_{2}<\ldots<\lambda_{s}
$$

be the exponentials of the positive Lyapunov exponents of $f$ over $\mathcal{L}$ (so we have $\left.1<\lambda_{1}\right)$. Fix an arbitrary constant $\alpha \in(0,1]$ such that

$$
\lambda_{j}^{\alpha}<\lambda_{j+1}, \quad 1 \leq j<s .
$$

Next, set $\nu_{0}=\left(1+\lambda_{1}\right) / 2, \nu_{1}=\lambda_{1}+\left(\lambda_{2}-\lambda_{1}\right) / 3$ and $\nu_{2}=\lambda_{1}+2\left(\lambda_{2}-\lambda_{1}\right) / 3$, so that $1<\nu_{0}<\lambda_{1}<\nu_{1}<\nu_{2}<\lambda_{2}$. Then take $\mu>0$ so small that

$$
\nu_{0} e^{8 \mu}<\lambda_{1}, \quad \nu_{2} e^{8 \mu}<\lambda_{2}
$$

(it then follows that $\lambda_{1} e^{8 \mu}<\nu_{1}$ and $\nu_{1} e^{8 \mu}<\nu_{2}$ ), and

$$
0<\mu<\min \left\{\frac{\alpha}{2(2+\alpha)} \ln \nu_{0}, \ln \frac{\lambda_{1}+\nu_{1}}{2 \lambda_{1}}, \ln \frac{2 \lambda_{2}}{\lambda_{2}+\nu_{2}}\right\} .
$$

Set

$$
\gamma=\max \left\{\left(\nu_{0} / \lambda_{1}\right)^{\alpha}, \nu_{1} / \nu_{2}\right\}<1,
$$

$\tilde{\mu}_{1}=\lambda_{1} e^{-2 \mu}, \mu_{1}=\lambda_{1} e^{-\mu}, \lambda_{1}^{\prime}=\lambda_{1} e^{\mu}, \tilde{\lambda}_{1}=\lambda_{1} e^{2 \mu}$ and $\mu_{2}=\lambda_{2} e^{-\mu}$. Then

$$
1<\nu_{0}<\tilde{\mu}_{1}<\mu_{1}<\lambda_{1}<\lambda_{1}^{\prime}<\tilde{\lambda}_{1}<\nu_{1}<\nu_{2}<\mu_{2}<\lambda_{2} .
$$

Fix for a moment $\mu>0$ with the above properties. Then, for $x \in \mathcal{L}$ we have an $f$-invariant decomposition $E^{u}(x)=E_{1}^{u}(x) \oplus E_{2}^{u}(x) \oplus \ldots \oplus E_{s}^{u}(x)$ into subspaces of constant dimensions $n_{1}, \ldots, n_{s}$ such that for some Lyapunov $\mu$-regularity function $R=R_{\mu}: \mathcal{L} \longrightarrow(1, \infty)$, i.e. a function with

$$
e^{-\mu} \leq \frac{R(f(x))}{R(x)} \leq e^{\mu}, \quad x \in \mathcal{L}
$$

we have

$$
\frac{1}{R(x) e^{n \mu}} \leq \frac{\left\|d f^{n}(x) \cdot v\right\|}{\lambda_{i}^{n}\|v\|} \leq R(x) e^{n \mu}, \quad x \in \mathcal{L}, v \in E_{i}^{u}(x) \backslash\{0\}, n \geq 0 .
$$

For $x \in \mathcal{L}$, set $\widetilde{E}_{2}^{u}(x)=E_{2}^{u}(x) \oplus \ldots \oplus E_{s}^{u}(x)$. For any $u \in E^{u}(x)$ we will write $u=u^{(1)}+u^{(2)}$, where $u^{(1)} \in E_{1}^{u}(x)$ and $u^{(2)} \in \widetilde{E}_{2}^{u}(x)$. We will denote by $\|\cdot\|$ the norm on $E^{u}(x)$ generated by the Riemann metric, and we will also use the norm $|u|=\max \left\{\left\|u^{(1)}\right\|,\left\|u^{(2)}\right\|\right\}$. Taking the regularity function $R(x)$ appropriately (see [P1], $\mathrm{BP}]$ or $[\mathrm{PS}]$ ), we may assume that

$$
|u| \leq\|u\| \leq R(x)|u|, \quad x \in \mathcal{L}, u \in E^{u}(x) .
$$

It follows from Pesin's theory (see [P1], $[\mathrm{BP}]$ ) or [PS]) that the invariant bundle $\left\{\widetilde{E}_{2}^{u}(x)\right\}_{x \in \mathcal{L}}$ is uniquely integrable over $\mathcal{L}$; i.e., there exists a continuous $f$-invariant family $\left\{W_{\tilde{q}(x)}^{u, 2}(x)\right\}_{x \in \mathcal{L}}$ of $C^{2}$ submanifolds $W_{\tilde{q}(x)}^{u, 2}(x)$ of $M$ tangent to the bundle $\widetilde{E}_{2}^{u}$ for some Lyapunov $\mu / 2$-regularity function $\tilde{q}=\tilde{q}_{\mu / 2}: \mathcal{L} \longrightarrow(0,1)$. Moreover, it follows from Theorem 6.6 in $[\mathrm{PS}$ and (3.1) that there exists an $f$-invariant family $\left\{W_{\tilde{q}(x)}^{u, 1}(x)\right\}_{x \in \mathcal{L}}$ of $C^{1+\alpha}$ submanifolds $W_{\tilde{q}(x)}^{u, 1}(x)$ of $M$ tangent to the bundle $\widetilde{E}_{1}^{u}$. (However this family is not unique in general.) For each $x \in \mathcal{L}$ fix an $f$-invariant family $\left\{W_{\tilde{q}(x)}^{u, 1}(x)\right\}_{x \in \mathcal{L}}$ with the latter properties. Then we can find a Lyapunov $\mu$ regularity function $q=q_{\mu}: \mathcal{L} \longrightarrow(0,1)$ and for any $x \in \mathcal{L}$ a $C^{1+\alpha}$ diffeomorphism

$$
\Phi_{x}: E^{u}(x ; q(x)) \longrightarrow \Phi_{x}\left(E^{u}(x ; q(x))\right) \subset W_{\tilde{q}(x)}^{u}(x)
$$


such that

$$
\Phi_{x}\left(E_{1}^{u}(x ; q(x))\right) \subset W_{\tilde{q}(x)}^{u, 1}(x), \quad \Phi_{x}\left(\widetilde{E}_{2}^{u}(x ; q(x))\right) \subset W_{\tilde{q}(x)}^{u, 2}(x) \quad x \in \mathcal{L} .
$$

We will assume without loss of generality that the regularity function $R$ satisfies

$$
\left\|d \Phi_{x}(u)\right\| \leq R(x), \quad\left\|\left(d \Phi_{x}(u)\right)^{-1}\right\| \leq R(x), \quad x \in \mathcal{L}, u \in E^{u}(x ; q(x)) .
$$

For any $x \in \mathcal{L}$ consider the $C^{1+\alpha}$ map (defined locally near 0 )

$$
\hat{f}_{x}=\left(\Phi_{f(x)}\right)^{-1} \circ f \circ \Phi_{x}: E^{u}(x) \longrightarrow E^{u}(f(x)) .
$$

Given $y \in \mathcal{L}$ and any integer $k \geq 1$ we will use the notation

$\hat{f}_{y}^{k}=\hat{f}_{f^{k-1}(y)} \circ \ldots \circ \hat{f}_{f(y)} \circ \hat{f}_{y}, \quad \hat{f}_{y}^{-k}=\left(\hat{f}_{f^{-k}(y)}\right)^{-1} \circ \ldots \circ\left(\hat{f}_{f^{-2}(y)}\right)^{-1} \circ\left(\hat{f}_{f^{-1}(y)}\right)^{-1}$, at any point where these sequences of maps are well-defined.

It is well known (see e.g. section 3 in $[\mathrm{PS}$, or section 8 in [LY]) that there exist Lyapunov $\mu$-regularity functions $\Gamma=\Gamma_{\mu}: \mathcal{L} \longrightarrow[1, \infty)$ and $q=q_{\mu}: \mathcal{L} \longrightarrow(0,1)$ and for each $x \in \mathcal{L}$ a norm $\|\cdot\|_{x}^{\prime}$ on $T_{x} M$ such that

$$
\|v\| \leq\|v\|_{x}^{\prime} \leq \Gamma(x)\|v\| \quad, \quad x \in \mathcal{L}, v \in T_{x} M,
$$

and for any $x \in \mathcal{L}$ and any integer $n \geq 0$, assuming $\hat{f}_{x}^{j}(u), \hat{f}_{x}^{j}(v) \in E^{u}\left(f^{j}(x)\right.$, $\left.q\left(f^{j}(x)\right)\right)$ are well-defined for all $j=1, \ldots, n$, the following hold:

$$
\mu_{2}^{n}\|u-v\|_{x}^{\prime} \leq\left\|\hat{f}_{x}^{n}(u)-\hat{f}_{x}^{n}(v)\right\|_{f^{n}(x)}^{\prime}, \quad u, v \in \widetilde{E}_{2}^{u}(x ; q(x)),
$$

$$
\mu_{1}^{n}\|u-v\|_{x}^{\prime} \leq\left\|\hat{f}_{x}^{n}(u)-\hat{f}_{x}^{n}(v)\right\|_{f^{n}(x)}^{\prime} \leq\left(\lambda_{1}^{\prime}\right)^{n}\|u-v\|_{x}^{\prime}, u, v \in E_{1}^{u}(x ; q(x)),
$$

$$
\begin{gathered}
\mu_{1}^{n}\|u-v\|_{x}^{\prime} \leq\left\|\hat{f}_{x}^{n}(u)-\hat{f}_{x}^{n}(v)\right\|_{f^{n}(x)}^{\prime}, \quad u, v \in E^{u}(x ; q(x)), \\
\mu_{1}^{n}\|v\|_{x}^{\prime} \leq\left\|d \hat{f}_{x}^{n}(0) \cdot v\right\|_{f^{n}(x)}^{\prime} \leq\left(\lambda_{1}^{\prime}\right)^{n}\|v\|_{x}^{\prime}, \quad v \in E_{1}^{u}(x),
\end{gathered}
$$

and

$$
\mu_{2}^{n}\|v\|_{x}^{\prime} \leq\left\|d \hat{f}_{x}^{n}(0) \cdot v\right\|_{f^{n}(x)}^{\prime}, \quad v \in \widetilde{E}_{2}^{u}(x) .
$$

Clearly each of the above inequalities provides a corresponding inequality involving the norm $\|\cdot\|$. For example (3.9) and (3.10) imply (under the assumptions above)

$$
\mu_{2}^{n}\|u-v\| \leq \mu_{2}^{n}\|u-v\|_{x}^{\prime} \leq\left\|\hat{f}_{x}^{n}(u)-\hat{f}_{x}^{n}(v)\right\|_{f^{n}(x)}^{\prime} \leq \Gamma\left(f^{n}(x)\right)\left\|\hat{f}_{x}^{n}(u)-\hat{f}_{x}^{n}(v)\right\|
$$

for all $x \in \mathcal{L}$ and all $u, v \in \widetilde{E}_{2}^{u}(x)$.

3.2. Balls in Bowen's metric. We will use the notation from section 3.1. Given $t>0$ and $\delta>0$ set

$$
B_{t}^{u}(x, \delta)=\left\{y \in W_{\delta}^{u}(x): d\left(\phi_{t}(x), \phi_{t}(y)\right) \leq \delta\right\} .
$$

Our aim in this section is to prove the following.

Theorem 3.1. For every $\epsilon>0$ there exist Lyapunov $\epsilon$-regularity functions $\omega$ : $\mathcal{L} \longrightarrow(0,1)$ and $G: \mathcal{L} \longrightarrow[1, \infty)$ such that for any $0<\delta_{1}<\delta_{2}$ there exists a constant $K=K\left(\delta_{1}, \delta_{2}\right) \geq 1$ with the following property: for all $x \in \mathcal{L}$ with $\delta_{2} \leq \omega(x)$ and all $t>0$ we have

$$
\operatorname{diam}\left(B_{t}^{u}\left(\phi_{-t}(x), \delta_{2}\right)\right) \leq K G(x) \operatorname{diam}\left(B_{t}^{u}\left(\phi_{-t}(x), \delta_{1}\right)\right) .
$$


Fix an arbitrary $\epsilon>0$. Let $\mu>0$ satisfy (3.2), (3.3) and $0<\mu<\frac{\epsilon \alpha}{12}$.

For a non-empty set $X \subset E^{u}(x)$ set

$$
\ell(X)=\sup \{\|u\|: u \in X\} .
$$

Given $z \in \mathcal{L}$ and $p \geq 1$, setting $x=f^{p}(z)$, define

$$
\begin{gathered}
\widehat{B}_{p}^{u}(z, \delta)=\left\{u \in E^{u}(z):\left\|\hat{f}_{z}^{p}(u)\right\| \leq \delta\right\}, \quad \widehat{B}_{p}^{u, 1}(z, \delta)=E_{1}^{u}(z) \cap \widehat{B}_{p}^{u}(z, \delta), \\
\widetilde{B}_{p}^{u}(z, \delta)=\left\{u \in E^{u}(z):\left\|d \hat{f}_{z}^{p}(0) \cdot u\right\| \leq \delta\right\}, \quad \widetilde{B}_{p}^{u, 1}(z, \delta)=E_{1}^{u}(z) \cap \widetilde{B}_{p}^{u}(z, \delta) .
\end{gathered}
$$

Notice that $u \in E_{1}^{u}(z)$ implies $\hat{f}_{z}^{p}(u) \in E_{1}^{u}\left(f^{p}(z)\right)$ whenever $\hat{f}_{z}^{p}(u)$ is well-defined.

Theorem 3.1 will be derived from Lemma 3.3 below and the following proposition.

Proposition 3.2. There exists a $12 \mu / \alpha$-regularity function $\omega: \mathcal{L} \longrightarrow(0,1)$ with $\omega(x) \leq q(x)$ for all $x \in \mathcal{L}$ and a $4 \mu$-regularity function $G: \mathcal{L} \longrightarrow[1, \infty)$ such that for any $x \in \mathcal{L}$, any $\delta \in(0, \omega(x)]$ and any integer $p \geq 1$ for $z=f^{-p}(x)$ we have $\ell\left(\widehat{B}_{p}^{u}(z, \delta)\right) \leq G(x) \ell\left(\widehat{B}_{p}^{u, 1}(z, \delta)\right)$.

The proof of Proposition 3.2 takes most of this section.

Taylor's formula (see also section 3 in $[$ PS ) implies that there exists a Lyapunov $\mu$-regularity function $D=D_{\mu}: \mathcal{L} \longrightarrow[1, \infty)$ such that for $x \in \mathcal{L}$ and $i= \pm 1$ we have

$$
\left\|\hat{f}_{x}^{i}(v)-\hat{f}_{x}^{i}(u)-d \hat{f}_{x}^{i}(u) \cdot(v-u)\right\| \leq D(x)\|v-u\|^{1+\alpha}, u, v \in E^{u}(x ; q(x)) .
$$

Fix for a moment $x \in \mathcal{L}$ and an integer $p \geq 1$, set $z=f^{-p}(x)$ and given $v \in E^{u}(z ; q(z))$, set

$$
z_{j}=f^{j}(z), \quad v_{j}=\hat{f}_{z}^{j}(v) \in E^{u}\left(z_{j}\right), \quad w_{j}=d \hat{f}_{z}^{j}(0) \cdot v \in E^{u}\left(z_{j}\right)
$$

for any $j=0,1, \ldots, p$ (assuming that these points are well-defined).

Lemma 3.3. There exist a Lyapunov $6 \mu$-regularity function $L: \mathcal{L} \longrightarrow[1, \infty)$ and a Lyapunov $7 \mu / \alpha$-regularity function $\tilde{r}: \mathcal{L} \longrightarrow(0,1)$ with $\tilde{r} \leq q$ such that for any $x \in \mathcal{L}$, any integer $p \geq 1$ and any $v \in E^{u}(z, \tilde{r}(z))$ with $\left\|\hat{f}_{z}^{p}(v)\right\| \leq \tilde{r}(x)$, where $z=f^{-p}(x)$, we have $\left\|w_{p}^{(1)}-v_{p}^{(1)}\right\| \leq L(x)\left|v_{p}\right|^{1+\alpha}$. Moreover, if $\left|v_{p}\right|=\left\|v_{p}^{(1)}\right\| \neq 0$, then $1 / 2 \leq\left\|w_{p}^{(1)}\right\| /\left\|v_{p}^{(1)}\right\| \leq 2$.

Proof of Lemma 3.3. One checks easily that

$$
\tilde{r}(x)=\left(\frac{1-\gamma}{2}\right)^{1 / \alpha} \cdot \frac{q(x)}{D(x)^{1 / \alpha} \Gamma(x)^{2 / \alpha+1} R(x)^{1 / \alpha+1}} \leq q(x)
$$

defines a Lyapunov $7 \mu / \alpha$-regularity function on $\mathcal{L}$.

Let $x \in \mathcal{L}$ and $z=f^{-p}(x)$ for some integer $p \geq 1$, and let $v \in E^{u}(z, \tilde{r}(z))$ be such that $\left\|\hat{f}_{z}^{p}(v)\right\| \leq \tilde{r}(x)$. Using the notation (3.17), by (3.9) and (3.12),

$$
\left\|v_{k}\right\|=\left\|\hat{f}_{z}^{k}(v)\right\| \leq \frac{\Gamma(x)}{\mu_{1}^{p-k}}\left\|\hat{f}_{z}^{p}(v)\right\|=\frac{\Gamma(x)}{\mu_{1}^{p-k}}\left\|v_{p}\right\|
$$

for all $k=0,1, \ldots, p-1$. It follows from (3.16) that $\left\|\hat{f}_{z}(v)-d \hat{f}_{z}(0) \cdot v\right\| \leq$ $D(z)\|v\|^{1+\alpha}$, so $w_{1}=\hat{f}_{z}(v)+u_{1}=v_{1}+u_{1}$ for some $u_{1} \in E^{u}\left(z_{1}\right)$ with $\left\|u_{1}\right\| \leq$ $D(z)\|v\|^{1+\alpha}$. Hence $w_{2}=d \hat{f}_{z_{1}}(0) \cdot w_{1}=d \hat{f}_{z_{1}}(0) \cdot v_{1}+d \hat{f}_{z_{1}}(0) \cdot u_{1}$. Using $(3.16)$ again, we get $d \hat{f}_{z_{1}}(0) \cdot v_{1}=\hat{f}_{z_{1}}\left(v_{1}\right)+u_{2}=v_{2}+u_{2}$ for some $u_{2} \in E^{u}\left(z_{2}\right)$ with 
$\left\|u_{2}\right\| \leq D\left(z_{1}\right)\left\|v_{1}\right\|^{1+\alpha}$. Thus, $w_{2}=v_{2}+u_{2}+d \hat{f}_{z_{1}}(0) \cdot u_{1}$. Continuing by induction, as in the proof of Lemma 3.3 in [St3, one derives

$$
w_{p}=v_{p}+u_{p}+d \hat{f}_{z_{p-1}}(0) \cdot u_{p-1}+d \hat{f}_{z_{p-2}}^{2}(0) \cdot u_{p-2}+\ldots+d \hat{f}_{z}^{p-1}(0) \cdot u_{1},
$$

where $u_{j} \in E^{u}\left(z_{j}\right)$ and $\left\|u_{j}\right\| \leq D\left(z_{j-1}\right)\left\|v_{j-1}\right\|^{1+\alpha}$ for all $j=1, \ldots, p$. Then

$$
\begin{gathered}
\left\|u_{j}\right\| \leq D\left(z_{j-1}\right)\left\|v_{j-1}\right\|^{1+\alpha} \leq \frac{D(x) e^{(p-j+1) \mu} \Gamma(x)^{1+\alpha}}{\mu_{1}^{(1+\alpha)(p-j+1)}}\left\|v_{p}\right\|^{1+\alpha} \\
\leq \frac{D(x) \Gamma(x)^{1+\alpha}}{\tilde{\mu}_{1}^{(1+\alpha)(p-j)}}\left\|v_{p}\right\|^{1+\alpha} .
\end{gathered}
$$

Combining the latter with (3.9) and (3.13) gives

$$
\left\|d \hat{f}_{z_{j}}^{p-j}(0) \cdot u_{j}^{(1)}\right\| \leq\left(\lambda_{1}^{\prime}\right)^{p-j} \Gamma\left(z_{j}\right)\left\|u_{j}\right\| \leq D(x) \Gamma(x)^{2+\alpha}\left(\frac{\tilde{\lambda}_{1}}{\tilde{\mu}_{1}^{1+\alpha}}\right)^{p-j}\left\|v_{p}\right\|^{1+\alpha}
$$

for all $j=1, \ldots, p$. Setting $L^{\prime}(x)=D(x) \Gamma(x)^{2+\alpha}$, and using (3.3) and (3.4) to get

$$
\frac{\tilde{\lambda}_{1}}{\tilde{\mu}_{1}^{1+\alpha}}=\frac{\lambda_{1} e^{2 \mu}}{\lambda_{1}^{1+\alpha} e^{-2 \mu(1+\alpha)}}=\frac{e^{2 \mu(2+\alpha)}}{\lambda_{1}^{\alpha}} \leq\left(\frac{\nu_{0}}{\lambda_{1}}\right)^{\alpha} \leq \gamma
$$

it follows that $\left\|d \hat{f}_{z_{j}}^{p-j}(0) \cdot u_{j}^{(1)}\right\| \leq L^{\prime}(x) \gamma^{p-j}\left\|v_{p}\right\|^{1+\alpha}$. Now (3.19) yields

$$
\left\|w_{p}^{(1)}-v_{p}^{(1)}\right\| \leq L^{\prime}(x)\left\|v_{p}\right\|^{1+\alpha} \sum_{j=1}^{p} \gamma^{p-j} \leq \frac{L^{\prime}(x)}{1-\gamma}\left\|v_{p}\right\|^{1+\alpha} \leq \frac{L^{\prime}(x) R(x)^{1+\alpha}}{1-\gamma}\left|v_{p}\right|^{1+\alpha} .
$$

Since $L(x)=L^{\prime}(x) R(x)^{1+\alpha} /(1-\gamma)$ is a Lyapunov $6 \mu$-regularity function, this proves the first part of the lemma.

If $\left|v_{p}\right|=\left\|v_{p}^{(1)}\right\|$, then the above gives $\left\|w_{p}^{(1)}-v_{p}^{(1)}\right\| \leq L(x)\left\|v_{p}^{(1)}\right\|^{1+\alpha}$, so

$$
\left|\frac{\left\|w_{p}^{(1)}\right\|}{\left\|v_{p}^{(1)}\right\|}-1\right| \leq L(x)\left\|v_{p}^{(1)}\right\|^{\alpha} \leq L(x)(\tilde{r}(x))^{\alpha} \leq \frac{1}{2},
$$

by the choice of $\tilde{r}(x)$. Hence $1 / 2 \leq\left\|w_{p}^{(1)}\right\| /\left\|v_{p}^{(1)}\right\| \leq 2$.

Corollary 3.4. Under the assumptions of Lemma 3.3, for $u=v^{(1)} \in E_{1}^{u}(z)$ we have $1 / 2 \leq\left\|w_{p}^{(1)}\right\| /\left\|u_{p}\right\| \leq 2$.

Proof. We just apply Lemma 3.3 replacing $v$ by $u$. Since $\hat{f}_{z}^{p}(u) \in E_{1}^{u}(x)$, we have $u_{p}=u_{p}^{(1)}$.

Lemma 3.5. Assume that the regularity function $r$ satisfies $r(x) \leq \tilde{r}(x)$ and

$$
r(x) \leq \min \left\{\left(\frac{1 / \nu_{2}-1 / \lambda_{2}}{2 e^{\mu} \Gamma^{2}(x) D(x)}\right)^{1 / \alpha},\left(\frac{1 / \lambda_{1}-1 / \nu_{1}}{2 e^{3 \mu} \Gamma^{2}(x) D(x)}\right)^{1 / \alpha}\right\}
$$

for all $x \in \mathcal{L}$. Then for any $x \in \mathcal{L}$ and any $V=V^{(1)}+V^{(2)} \in E^{u}(x ; r(x))$ we have

$$
\left\|\left(\hat{f}_{x}^{-1}\right)^{(2)}(V)\right\|_{f^{-1}(x)}^{\prime} \leq \frac{\left\|V^{(2)}\right\|_{x}^{\prime}}{\nu_{2}}
$$

and

$$
\left\|\left(\hat{f}_{x}^{-1}\right)^{(1)}(V)\right\|_{f^{-1}(x)}^{\prime} \geq \frac{\left\|V^{(1)}\right\|_{x}^{\prime}}{\nu_{1}}
$$


Proof of Lemma 3.5. Let $x \in \mathcal{L}, y=f^{-1}(x)$ and let $V=V^{(1)}+V^{(2)} \in E^{u}(x ; r(x))$. By (3.16),

$$
\hat{f}_{x}^{-1}(V)-\hat{f}_{x}^{-1}\left(V^{(1)}, 0\right)=d \hat{f}_{x}^{-1}\left(V^{(1)}, 0\right) \cdot\left(0, V^{(2)}\right)+\xi
$$

for some $\xi \in E^{u}(y)$ with $\|\xi\| \leq D(y)\left\|V^{(2)}\right\|^{1+\alpha}$. Using $\left\|V^{(2)}\right\| \leq r(x)$ and (3.9), the latter gives $\|\xi\|_{y}^{\prime} \leq \Gamma(y)\|\xi\| \leq \Gamma(y) D(y)\left\|V^{(2)}\right\|^{1+\alpha} \leq \Gamma(x) D(x) e^{2 \mu}\left\|V^{(2)}\right\|^{1+\alpha}$, so $\|\xi\|_{y}^{\prime} \leq \Gamma^{2}(x) D(x) e^{2 \mu}\left\|V^{(2)}\right\|_{x}^{\prime} r^{\alpha}(x)$. Since $\hat{f}_{x}^{-1}\left(V^{(1)}, 0\right) \in E_{1}^{u}(y)$, it follows from (3.14) that

$$
\begin{aligned}
\left\|\left(\hat{f}_{x}^{-1}\right)^{(2)}(V)\right\|_{y}^{\prime} & \leq\left\|d \hat{f}_{x}^{-1}\left(V^{(1)}, 0\right) \cdot\left(0, V^{(2)}\right)\right\|_{y}^{\prime}+\|\xi\|_{y}^{\prime} \\
& \leq\left\|V^{(2)}\right\|_{x}^{\prime}\left(\frac{1}{\mu_{2}}+\Gamma^{2}(x) D(x) e^{2 \mu} r^{\alpha}(x)\right) .
\end{aligned}
$$

Now (3.9) and (3.20) imply that

$$
\frac{1}{\mu_{2}}+\Gamma^{2}(x) D(x) e^{2 \mu} r^{\alpha}(x) \leq \frac{e^{\mu}}{\lambda_{2}}+e^{\mu} \frac{1 / \nu_{2}-1 / \lambda_{2}}{2}=e^{\mu} \frac{\lambda_{2}+\nu_{2}}{2 \lambda_{2} \nu_{2}}<\frac{1}{\nu_{2}},
$$

since by (3.3) we have $e^{\mu}<\frac{2 \lambda_{2}}{\lambda_{2}+\nu_{2}}$. The above and (3.23) imply (3.21).

Similarly, we have

$$
\hat{f}_{x}^{-1}(V)-\hat{f}_{x}^{-1}\left(0, V^{(2)}\right)=d \hat{f}_{x}^{-1}\left(0, V^{(2)}\right) \cdot\left(V^{(1)}, 0\right)+\eta
$$

for some $\eta \in E^{u}(y)$ with $\|\eta\| \leq D(y)\left\|V^{(1)}\right\|^{1+\alpha}$. As before we get

$$
\|\eta\|_{y}^{\prime} \leq \Gamma^{2}(x) D(x) e^{2 \mu}\left\|V^{(1)}\right\|_{x}^{\prime} r^{\alpha}(x) .
$$

Since $\hat{f}_{x}^{-1}\left(0, V^{(2)}\right) \in \widetilde{E}_{2}^{u}(y)$, by $(3.13)$,

$$
\begin{aligned}
\left\|\left(\hat{f}_{x}^{-1}\right)^{(1)}(V)\right\|_{y}^{\prime} & \geq\left\|d \hat{f}_{x}^{-1}\left(0, V^{(2)}\right) \cdot\left(V^{(1)}, 0\right)\right\|_{y}^{\prime}-\|\eta\|_{y}^{\prime} \\
& \geq\left\|V^{(1)}\right\|_{x}^{\prime}\left(\frac{1}{\lambda_{1}^{\prime}}-\Gamma^{2}(x) D(x) e^{2 \mu} r^{\alpha}(x)\right) .
\end{aligned}
$$

Now (3.20) implies that

$$
\frac{1}{\lambda_{1}^{\prime}}-\Gamma^{2}(x) D(x) e^{2 \mu} r^{\alpha}(x) \geq \frac{1}{\lambda_{1} e^{\mu}}-\frac{1 / \lambda_{1}-1 / \nu_{1}}{2 e^{\mu}}=\frac{\lambda_{1}+\nu_{1}}{2 e^{\mu} \lambda_{1} \nu_{1}}>\frac{1}{\nu_{1}},
$$

since by (3.3) we have $e^{\mu}<\frac{\lambda_{1}+\nu_{1}}{2 \lambda_{1}}$. The above and (3.24) imply (3.22).

Proof of Proposition 3.2. Define $\omega: \mathcal{L} \longrightarrow(0,1)$ by

$$
\omega(x)=\left(\frac{1-\gamma}{2}\right)^{1 / \alpha} \frac{q(x)}{16 \nu_{1} \Gamma(x)^{2 / \alpha+3} R(x)^{1 / \alpha+1}}\left(\frac{1 / \nu_{2}-1 / \lambda_{2}}{2 e^{3 \mu} D(x)}\right)^{1 / \alpha} .
$$

Clearly $\omega(x) \leq \tilde{r}(x)$, the function defined by (3.18). Moreover, $1 / \lambda_{1}-1 / \nu_{1}>$ $1 / \nu_{2}-1 / \lambda_{2}$ shows that $r(x)=16 \nu_{1} \Gamma^{3}(x) R(x) \omega(x)$ satisfies (3.20), so Lemma 3.5 applies. It is easy to check that $\omega$ is a Lyapunov $12 \mu / \alpha$-regularity function.

Let $x \in \mathcal{L}$ and let $p \geq 1$ be an integer. Set $z=f^{-p}(x)$. Given $\delta>0$ with $\delta \leq \omega(x)$, we have $16 \nu_{1} \Gamma^{3}(x) \delta \leq r(x)$. Let $v \in \widehat{B}_{p}^{u}(z, \delta)$ be such that $\|v\|$ is the maximum possible. Then we must have $\left\|v_{p}\right\|=\delta$, where we use the notation in (3.17). Set $V=v_{p}$.

Next, consider two cases. 
Case 1. $\left\|v^{(2)}\right\| \geq\left\|v^{(1)}\right\|$. Let $U=\left(U^{(1)}, 0\right)$ be such that $U^{(1)} \in E_{1}^{u}(x)$ is an arbitrary element with $\left\|U^{(1)}\right\|=\delta$. Then $u=\hat{f}_{x}^{-p}(U) \in \widehat{B}_{p}^{u, 1}(z, \delta)$, and applying (3.22) $p$ times gives $\|u\|_{z}^{\prime} \geq \frac{\left\|U^{(1)}\right\|_{x}^{\prime}}{\nu_{1}^{p}} \geq \frac{\delta}{\nu_{1}^{p}}$. Similarly, applying (3.21) $p$ times and using (3.4) implies that

$$
\left\|v^{(2)}\right\|_{z}^{\prime} \leq \frac{\left\|V^{(2)}\right\|_{x}^{\prime}}{\nu_{2}^{p}} \leq \frac{\Gamma(x)\left\|V^{(2)}\right\|}{\nu_{2}^{p}} \leq \Gamma(x) \frac{\delta}{\nu_{2}^{p}} \leq \Gamma(x) \gamma^{p} \frac{\delta}{\nu_{1}^{p}} \leq \Gamma(x) \gamma^{p}\|u\|_{z}^{\prime} .
$$

This and (3.9) give

$\left\|v^{(2)}\right\| \leq\left\|v^{(2)}\right\|_{z}^{\prime} \leq \Gamma(x) \gamma^{p}\|u\|_{z}^{\prime} \leq \Gamma(x) \gamma^{p} \Gamma(z)\|u\| \leq \Gamma(x) \gamma^{p} e^{p \mu} \Gamma(x)\|u\| \leq \Gamma^{2}(x)\|u\|$. The latter yields $\left\|v^{(1)}\right\| \leq\left\|v^{(2)}\right\| \leq\left\|v^{(2)}\right\|_{z}^{\prime} \leq \Gamma^{2}(x)\|u\|$, and therefore $|v| \leq$ $\Gamma^{2}(x)\|u\|$. Hence $\|v\| \leq R(x)|v| \leq \Gamma^{2}(x) R(x)\|u\|$, which shows that $\ell\left(\widehat{B}_{p}^{u}(z, \delta)\right) \leq$ $\Gamma^{2}(x) R(x) \ell\left(\widehat{B}_{p}^{u, 1}(z, \delta)\right)$.

Case 2. $\left\|v^{(2)}\right\|<\left\|v^{(1)}\right\|$. Set $x_{j}=z_{p-j}=f^{-j}(x)$. Let $q<p$ be the largest integer with $\left\|\left(\hat{f}_{x}^{-q}\right)^{(2)}(V)\right\| \geq\left\|\left(\hat{f}_{x}^{-q}\right)^{(1)}(V)\right\|$. Set $\tilde{p}=p-q, \tilde{x}=x_{\tilde{p}}, u=\left(v^{(1)}, 0\right) \in E_{1}^{u}(z)$ and $u_{j}=\hat{f}_{z}^{j}(u)$. Then clearly $\|u\|=\left\|v^{(1)}\right\|$, and by the choice of $q,\left\|v_{\tilde{p}}^{(2)}\right\| \leq\left\|v_{\tilde{p}}^{(1)}\right\|$. Thus, $\left|v_{\tilde{p}}\right|=\left\|v_{\tilde{p}}^{(1)}\right\|$, and now it follows from Lemma 3.3 and Corollary 3.4 with $p$ replaced by $\tilde{p}$ that $\left\|u_{\tilde{p}}\right\| \leq 4\left\|v_{\tilde{p}}^{(1)}\right\|$, so $\left\|u_{\tilde{p}}\right\|_{\tilde{x}}^{\prime} \leq 4 \Gamma(\tilde{x})\left\|v_{\tilde{p}}^{(1)}\right\|_{\tilde{x}}^{\prime}$. Again by the choice of $q,\left\|v_{\tilde{p}+1}^{(2)}\right\| \geq\left\|v_{\tilde{p}+1}^{(1)}\right\|$, so

$$
\left\|v_{\tilde{p}+1}^{(1)}\right\|_{x_{\tilde{p}+1}}^{\prime} \leq \Gamma\left(x_{\tilde{p}+1}\right)\left\|v_{\tilde{p}+1}^{(1)}\right\| \leq \Gamma\left(x_{\tilde{p}+1}\right)\left\|v_{\tilde{p}+1}^{(2)}\right\| \leq \Gamma(x) e^{q \mu}\left\|v_{\tilde{p}+1}^{(2)}\right\|_{x_{\tilde{p}+1}}^{\prime} .
$$

Hence, using (3.12), (3.11), (3.21) and (3.9), we get

$$
\begin{aligned}
\left\|u_{\tilde{p}+1}\right\|_{x_{\tilde{p}+1}}^{\prime} & \leq \lambda_{1}^{\prime}\left\|u_{\tilde{p}}\right\|_{\tilde{x}}^{\prime} \leq 4 \lambda_{1}^{\prime} \Gamma(\tilde{x})\left\|v_{\tilde{p}}^{(1)}\right\|_{\tilde{x}}^{\prime} \leq \frac{4 \lambda_{1}^{\prime}}{\mu_{1}} \Gamma(\tilde{x})\left\|v_{\tilde{p}+1}^{(1)}\right\|_{x_{\tilde{p}+1}}^{\prime} \\
& \leq 4 \lambda_{1}^{\prime} \Gamma^{2}(x) e^{2 q \mu}\left\|v_{\tilde{p}+1}^{(2)}\right\|_{x_{\tilde{p}+1}}^{\prime} \leq \frac{4 \lambda_{1}^{\prime}}{\nu_{2}^{q-1}} \Gamma^{2}(x) e^{2 q \mu}\left\|V^{(2)}\right\|_{x}^{\prime} .
\end{aligned}
$$

Thus, $\left\|u_{\tilde{p}+1}\right\|_{x_{\tilde{p}+1}}^{\prime} \leq \frac{4 \lambda_{1}^{\prime} \Gamma^{3}(x) e^{2 q \mu} \delta}{\nu_{2}^{q-1}}$. Now (3.11) and (3.4) imply that

$$
\left\|u_{p}\right\|_{x}^{\prime} \leq\left(\lambda_{1}^{\prime}\right)^{q-1}\left\|u_{\tilde{p}+1}\right\|_{x_{\tilde{p}+1}}^{\prime} \leq\left(\lambda_{1}^{\prime}\right)^{q-1} \frac{4 \lambda_{1}^{\prime} \Gamma^{3}(x) e^{2 q \mu} \delta}{\nu_{2}^{q-1}} \leq 4 \nu_{1} \Gamma^{3}(x) \delta \gamma^{q-1},
$$

and, using $\gamma<1$ and (3.9), we get $\left\|u_{p}\right\| \leq 4 \nu_{1} \Gamma^{3}(x) \delta$. Thus, $u \in \widehat{B}_{p}^{u, 1}\left(z, 4 \nu_{1} \Gamma^{3}(x) \delta\right)$.

Since $\|u\|=\left\|v^{(1)}\right\|>\left\|v^{(2)}\right\|$, it follows that $\|u\| \geq|v|$, so

$$
\ell\left(\widehat{B}_{p}^{u}(z, \delta)\right)=\|v\| \leq R(x)|v| \leq R(x)\|u\| \leq R(x) \ell\left(\widehat{B}_{p}^{u, 1}\left(z, 4 \nu_{1} \Gamma^{3}(x) \delta\right)\right) .
$$

On the other hand, it follows from Lemma 3.3, Corollary 3.4 and the linearity of the map $d \hat{f}_{z}^{p}(0)$ that $\ell\left(\widehat{B}_{p}^{u, 1}\left(z, 4 \nu_{1} \Gamma^{3}(x) \delta\right)\right) \leq 16 \nu_{1} \Gamma^{3}(x) \ell\left(\widehat{B}_{p}^{u, 1}(z, \delta)\right)$. Thus, $\ell\left(\widehat{B}_{p}^{u}(z, \delta)\right) \leq 16 \nu_{1} \Gamma^{3}(x) R(x) \ell\left(\widehat{B}_{p}^{u, 1}(z, \delta)\right)$.

It follows from cases 1 and 2 that the regularity functions $\omega(x)$ and $G(x)=$ $16 \nu_{1} \Gamma^{3}(x) R(x)$ satisfy the requirements of the proposition.

Proof of Theorem 3.1. Clearly it is enough to prove the analogous statement for sets of the form $\widehat{B}_{p}^{u}(z, \delta)$, and instead of diameters it is enough to work with $\ell(\cdot)$.

Assume that the regularity functions $\omega$ and $G(x)$ are as in Proposition 3.2. 
Let $x \in \mathcal{L}, p \geq 1$ be an integer and $z=f^{-p}(x)$. We will use again the notation (3.17). Let $0<\delta_{1}<\delta_{2} \leq \omega(x)$. It follows from Proposition 3.2 that $\ell\left(\widehat{B}_{p}^{u}\left(z, \delta_{2}\right)\right) \leq G(x) \ell\left(\widehat{B}_{p}^{u, 1}\left(z, \delta_{2}\right)\right)$, while the second part of Lemma 3.3 shows that $\ell\left(\widehat{B}_{p}^{u, 1}\left(z, \delta_{2}\right)\right) \leq \ell\left(\widetilde{B}_{p}^{u, 1}\left(z, 2 \delta_{2}\right)\right)$. Next, $\ell\left(\widetilde{B}_{p}^{u, 1}\left(z, 2 \delta_{2}\right)\right)=\frac{4 \delta_{2}}{\delta_{1}} \ell\left(\widetilde{B}_{p}^{u, 1}\left(z, \delta_{1} / 2\right)\right)$ by the linearity of the map $d \hat{f}_{z}^{p}(0)$. Then using again the second part of Lemma 3.3, we get $\ell\left(\widetilde{B}_{p}^{u, 1}\left(z, \delta_{1} / 2\right)\right) \leq \ell\left(\widehat{B}_{p}^{u, 1}\left(z, \delta_{1}\right)\right) \leq \ell\left(\widehat{B}_{p}^{u}\left(z, \delta_{1}\right)\right)$. Combining all these inequalities gives $\ell\left(\widehat{B}_{p}^{u}\left(z, \delta_{2}\right)\right) \leq K G(x) \ell\left(\widehat{B}_{p}^{u}\left(z, \delta_{1}\right)\right)$, where $K=\frac{4 \delta_{2}}{\delta_{1}}$.

Proof of Theorem 1.1. Consider the Anosov flow $\psi_{t}=\phi_{-t}$ on $M$. Clearly this flow has the same set $\mathcal{L}$ of Lyapunov regular points. Let $\omega$ and $G$ be Lyapunov regular functions satisfying the requirements of Theorem 3.1 for the flow $\psi_{t}$. We will denote by $\mathcal{W}_{\delta}^{s}(x)$ and $\mathcal{W}_{\delta}^{u}(x)$ the local stable and unstable manifolds for the flow $\psi_{t}$. Clearly $W_{\delta}^{s}(x)=\mathcal{W}_{\delta}^{u}(x)$ and $W_{\delta}^{u}(x)=\mathcal{W}_{\delta}^{s}(x)$.

Given $x \in \mathcal{L}, t>0$ and $0<\delta_{1}<\delta_{2} \leq \omega(x)$, set $x^{\prime}=\phi_{t}(x)$, and notice that $\phi_{t}\left(B^{s}\left(x, \delta_{i}\right)\right)=\mathcal{B}_{t}^{u}\left(x^{\prime}, \delta_{i}\right)$ for $i=1,2$, where

$$
\mathcal{B}_{t}^{u}\left(x^{\prime}, \delta_{i}\right)=\left\{y^{\prime} \in \mathcal{W}_{\delta_{i}}^{u}\left(x^{\prime}\right): d\left(\psi_{t}\left(x^{\prime}\right), \psi_{t}\left(y^{\prime}\right)\right) \leq \delta_{i}\right\}
$$

Using Theorem 3.1 for the flow $\psi$, it follows that there exists a constant $K\left(\delta_{1}, \delta_{2}\right) \geq$ 1 such that $\operatorname{diam}\left(\mathcal{B}_{t}^{u}\left(x^{\prime}, \delta_{2}\right)\right) \leq K G(x) \operatorname{diam}\left(\mathcal{B}_{t}^{u}\left(x^{\prime}, \delta_{1}\right)\right)$, i.e. $\operatorname{diam}\left(\phi_{t}\left(B^{s}\left(x, \delta_{2}\right)\right)\right) \leq$ $K G(x) \operatorname{diam}\left(\phi_{t}\left(B^{s}\left(x, \delta_{1}\right)\right)\right)$.

\section{Decay of Cylinder diameters in a Markov Coding}

Again let $\mathcal{R}=\left\{R_{i}\right\}_{i=1}^{k}$ be a fixed Markov family as in section 2. Define the matrix $A=\left(A_{i j}\right)_{i, j=1}^{k}$ by $A_{i j}=1$ if $\mathcal{P}\left(\operatorname{Int}\left(R_{i}\right)\right) \cap \operatorname{Int}\left(R_{j}\right) \neq \emptyset$ and $A_{i j}=0$ otherwise. According to $[\mathrm{BR}$ ] (see section 2 there), we may assume that $\mathcal{R}$ is chosen in such a way that $A^{M_{0}}>0$ (all entries of the $M_{0}$-fold product of $A$ by itself are positive) for some integer $M_{0}>0$. In what follows we assume that the matrix $A$ has this property.

Given a finite string $\imath=\left(i_{0}, i_{1}, \ldots, i_{m}\right)$ of integers $i_{j} \in\{1, \ldots, k\}$, we will say that $\imath$ is admissible if for any $j=0,1, \ldots, m-1$ we have $A_{i_{j} i_{j+1}}=1$. Given an admissible string $\imath$, denote by $\stackrel{\circ}{C}[\imath]$ the set of those $x \in U$ so that $\sigma^{j}(x) \in \operatorname{Int}^{u}\left(U_{i_{j}}\right)$ for all $j=0,1, \ldots, m$. The set $C[\imath]=\overline{\stackrel{\circ}{C}[\imath]} \subset \Lambda$ will be called a cylinder of length $m$ in $U$, while $\stackrel{\circ}{C}[\imath]$ will be called an open cylinder of length $m$. It follows from the properties of the Markov family that $\stackrel{\circ}{C}[\imath]$ is an open dense subset of $C[\imath]$. Any cylinder of the form $C\left[i_{0}, i_{1}, \ldots, i_{m}, i_{m+1}, \ldots, i_{m+q}\right]$ will be called a subcylinder of $C[\imath]$ of co-length $q$.

In what follows the cylinders considered are always defined by finite admissible strings. Given $x \in U_{i}$ for some $i$ and $r>0$ we will denote by $B_{U}(x, r)$ the set of all $y \in U_{i}$ with $d(x, y)<r$.

It is easy to see that $\operatorname{diam}(C[\imath]) \rightarrow 0$ exponentially fast as $m \rightarrow \infty$. A much more subtle question is if there exists a constant $\rho \in(0,1)$ such that for any cylinder $\mathcal{C}=C\left[i_{0}, i_{1}, \ldots, i_{m}\right]$ and any subcylinder $\mathcal{C}^{\prime}=C\left[i_{0}, i_{1}, \ldots, i_{m}, i_{m+1}\right]$ we have $\operatorname{diam}\left(\mathcal{C}^{\prime}\right) \geq \rho \operatorname{diam}(\mathcal{C})$. Using Theorem 1.1 here we show that this is always the case under some regularity assumptions about the flow. 
Recall the constants $c_{0} \in(0,1)$ and $\gamma_{1}>\gamma>1$ from section 2 , and fix an integer $p_{1} \geq 1$ with

$$
\rho_{0}=\frac{1}{c_{0} \gamma^{p_{1}}}<\min \left\{\frac{\operatorname{diam}\left(U_{i}\right)}{\operatorname{diam}\left(U_{j}\right)}: i, j=1, \ldots, k\right\}
$$

Then clearly $\rho_{0}<1$. Set $\rho_{1}=\rho_{0}^{1 / p_{1}}$ and fix a constant $r_{0}>0$ with $2 r_{0}<$ $\min \left\{\operatorname{diam}\left(U_{i}\right): i=1, \ldots, k\right\}$ and for each $i=1, \ldots, k$ a point $\hat{z}_{i} \in \widehat{U}_{i}$ such that $B_{U}\left(\hat{z}_{i}, r_{0}\right) \subset \operatorname{Int}^{u}\left(U_{i}\right)$.

The following is an easy consequence of (2.1).

Lemma 4.1 (St1]). There exists a global constant $C_{1}>0$ such that for any cylinder $C[\imath]$ of length $m$ we have $\operatorname{diam}(C[\imath]) \leq C_{1} \rho_{1}^{m}$ and $\operatorname{diam}(C[\imath]) \geq \frac{c_{0} r_{0}}{\gamma_{1}^{m}}$.

In what follows we will assume that $\rho_{1} \in(0,1)$ and $C_{1}>0$ are fixed constants with the above property. Fix a constant $\epsilon>0$ such that

$$
e^{-\epsilon}>\sqrt{\rho_{1}} \text {. }
$$

From now on we will assume that the local stable holonomy maps through $\Lambda$ are uniformly Lipschitz. Then there exists a constant $L \geq 1$ such that $d\left(\pi_{y}(z), \pi_{y}\left(z^{\prime}\right)\right) \leq$ $L d\left(z, z^{\prime}\right)$ for all $x, y \in M$ with $d(x, y)<\epsilon_{1}$ and $z, z^{\prime} \in W_{\epsilon_{1}}^{u}(x)$. (See section 2 for the choice of $\epsilon_{1}$.)

Given $i=1, \ldots, k$, according to the choice of the Markov family $\left\{R_{i}\right\}$, the projection $\operatorname{pr}_{R_{i}}: W_{i}=\phi_{[-\chi, \chi]}\left(R_{i}\right) \longrightarrow R_{i}$ along the flow $\phi_{t}$ is well-defined and Lipschitz. Since the projection $\pi_{i}: R_{i} \longrightarrow U_{i}$ along stable leaves is Lipschitz, the map $\psi_{i}=\pi_{i} \circ \operatorname{pr}_{R_{i}}: W_{i} \longrightarrow W_{R_{i}}^{u}\left(z_{i}\right)$ is also Lipschitz. Thus, we may assume that the constant $L \geq 1$ is chosen sufficiently large so that $d\left(\psi_{i}(u), \psi_{i}(v)\right) \leq L d(u, v)$ for all $u, v \in W_{i}$ and all $i=1, \ldots, k$.

Next, if $V=W_{R}^{u}(x)$ is the unstable leaf of some point $x \in R_{i}$ and $\imath=\left(i_{0}=\right.$ $\left.i, i_{1}, \ldots, i_{m}\right)$ is an admissible sequence, consider the generalized cylinder

$$
C_{V}[\imath]=\left\{y \in V: \mathcal{P}^{j}(y) \in R_{i_{j}}, j=0,1, \ldots, m\right\} .
$$

Clearly, $\pi_{i}\left(C_{V}[\imath]\right)=C[\imath]$, so

$$
\frac{1}{L} \operatorname{diam}\left(C_{V}[\imath]\right) \leq \operatorname{diam}(C[\imath]) \leq L \operatorname{diam}\left(C_{V}[\imath]\right)
$$

for any choice of $V$ and the admissible sequence $\imath$. For $V$ as above, $x \in V$ and $\delta>0$ set $B_{V}(x, \delta)=\{y \in V: d(x, y)<\delta\}$.

Theorem 4.2. Assume that $\phi_{t}: M \longrightarrow M$ is a $C^{2}$ Anosov flow such that the local stable holonomy maps are uniformly Lipschitz. Then there exist a constant $\rho \in(0,1)$ and a positive integer $p_{0} \geq 1$ such that:

(a) For any cylinder $C[\imath]=C\left[i_{0}, \ldots, i_{m}\right]$ and any subcylinder $C\left[\imath^{\prime}\right]=C\left[i_{0}, i_{1}\right.$, $\left.\ldots, i_{m+1}\right]$ of $C[\imath]$ of co-length 1 we have $\rho \operatorname{diam}(C[\imath]) \leq \operatorname{diam}\left(C\left[\imath^{\prime}\right]\right)$.

(b) For any cylinder $C[\imath]=C\left[i_{0}, \ldots, i_{m}\right]$ and any subcylinder $C\left[\imath^{\prime}\right]=C\left[i_{0}, i_{1}\right.$, $\left.\ldots, i_{m+1}, \ldots, i_{m+p_{0}}\right]$ of co-length $p_{0}$ we have $\operatorname{diam}\left(C\left[\imath^{\prime}\right]\right) \leq \rho \operatorname{diam}(C[\imath])$.

Proof of Theorem 4.2. Notice that $\sigma^{m}\left(\widehat{C}\left[i_{0}, \ldots, i_{m}\right]\right)=\widehat{U}_{i_{m}}$.

As in section 2 we will assume that the point $z_{i} \in \operatorname{Int}\left(R_{i}\right)$ is Lyapunov regular. Given $\epsilon>0$ with $(4.2)$, let $\omega: \mathcal{L} \longrightarrow(0,1)$ and $G: \mathcal{L} \longrightarrow[1, \infty)$ be Lyapunov 
$\epsilon$-regularity functions with the properties described in Theorem 3.1. Fix a constant $r>0$ with $r \leq \min _{1 \leq i \leq k} \omega\left(z_{i}\right)$ such that $B_{U}\left(z_{i}, r\right) \subset \operatorname{Int}^{u}\left(U_{i}\right)$ for all $i=1, \ldots, k$, and set $G_{0}=\max _{1 \leq i \leq k} G\left(z_{i}\right)$. Then fix an integer $p \geq p_{1}$ so large that

$$
\rho_{1}^{(p+1) / 2}<\frac{r}{C_{1} L} .
$$

First note the following. Let $x \in R_{j}$ be such that $\mathcal{P}^{p+1}(x)=z_{i}$ for some $j$ and $i$. Then $z \in \mathcal{C}=C_{V}[\imath]$ for some $\imath=\left[i_{0}, \ldots, i_{p+1}\right]$ with $i_{0}=j$ and $i_{p+1}=i$, where $V=W_{R}^{u}(z)$. We claim that

$$
C_{V}[l] \subset B_{V}(z, \omega(x)) \subset V .
$$

Indeed, by Lemma $4.1, \operatorname{diam}(\mathcal{C}) \leq C_{1} \rho_{1}^{p+1}$. On the other hand, $\omega$ is a Lyapunov $\epsilon$-regularity function, so using (4.2), Lemma 4.1 and (4.3), we get

$$
\omega(x) \geq \omega\left(z_{i}\right) e^{-(p+1) \epsilon} \geq r \rho_{1}^{(p+1) / 2}=\frac{r}{\rho_{1}^{(p+1) / 2}} \rho_{1}^{p+1}>C_{1} L \rho_{1}^{p+1} \geq \operatorname{diam}\left(C_{V}[\imath]\right) .
$$

This proves (4.5).

(a) Assume that $m>p$, and let $\imath=\left[i_{0}, i_{1}, \ldots, i_{m}\right]$ and $\imath^{\prime}=\left[i_{0}, i_{1}, \ldots, i_{m}, i_{m+1}\right]$ be admissible sequences. Let $z \in R_{i_{0}}$ be such that $\mathcal{P}^{m+1}(z)=z_{i_{m+1}}$ and $\mathcal{P}^{j}(z) \in$ $R_{i_{j}}$ for all $j=0,1, \ldots, m+1$. Set $V=W_{R}^{u}(z), \mathcal{C}=C_{V}[\imath]$ and $\mathcal{C}^{\prime}=C_{V}\left[\imath^{\prime}\right]$.

Next, set $x=\mathcal{P}^{m-p}(z)$ and $V^{\prime}=W_{R}^{u}(x)$, and consider the cylinders $\widetilde{\mathcal{C}}=$ $C_{V^{\prime}}\left[i_{m-p}, i_{m-p+1}, \ldots, i_{m}\right]$ and $\widetilde{\mathcal{C}^{\prime}}=C_{V^{\prime}}\left[i_{m-p}, i_{m-p+1}, \ldots, i_{m}, i_{m+1}\right]$. Since $\mathcal{P}^{p+1}(x)$ $=z_{i_{m+1}}$, using (4.5) we get $\widetilde{\mathcal{C}} \subset B_{V^{\prime}}(x, \omega(x))$, and moreover $\widetilde{\mathcal{C}} \subset B_{V^{\prime}}\left(x, C_{1} \rho_{1}^{p+1}\right)$. On the other hand it is easy to see using (2.1) that $\widetilde{\mathcal{C}}^{\prime} \supset B_{V^{\prime}}\left(x, c_{0} r / \gamma_{1}^{p+1}\right)$.

We will now use Theorem 3.1 with $x$ and $z$ as above, $t=\tau_{m-p}(z)>0$ and

$$
0<\delta_{1}=\frac{c_{0} r}{L \gamma_{1}^{p+1}}<\delta_{2}=C_{1} L \rho_{1}^{p+1}<\omega(x) .
$$

By Theorem 3.1, there exists a constant $K=K\left(\delta_{1}, \delta_{2}\right)>0$ (depending on $\delta_{1}$ and $\delta_{2}$ which are constants in our case) such that

$$
\operatorname{diam}\left(B_{t}^{u}\left(z, \delta_{1}\right)\right) \geq \frac{1}{K G\left(z_{i_{m+1}}\right)} \operatorname{diam}\left(B_{t}^{u}\left(z, \delta_{2}\right)\right) \geq \frac{1}{K G_{0}} \operatorname{diam}\left(B_{t}^{u}\left(z, \delta_{2}\right)\right) .
$$

However, using the above information about $\widetilde{\mathcal{C}}$ and $\widetilde{\mathcal{C}^{\prime}}$, as in the proof of Proposition 3.3 in St1, one easily observes that $\mathcal{C}^{\prime} \supset B_{t}^{u}\left(z, \delta_{1}\right)$ and $\mathcal{C} \subset B_{t}^{u}\left(z, \delta_{2}\right)$. Thus, $\operatorname{diam}\left(\mathcal{C}^{\prime}\right) \geq \frac{1}{K G_{0}} \operatorname{diam}(\mathcal{C})$. Combining the latter with (4.3) gives $\operatorname{diam}\left(C\left[\imath^{\prime}\right]\right) \geq$ $\frac{1}{L^{2} K G_{0}} \operatorname{diam}(C[\imath])$.

This proves part (a) for $m>p$. Since there are only finitely many cylinders of length $\leq p$, it follows immediately that there exists $\rho \in\left(0,1 /\left(L^{2} K G_{0}\right)\right]$ which satisfies the requirements of part (a).

(b) This is derived combining a simple modification of the proof of Proposition 3.3(b) in St1] with an argument similar to the above. We omit the details.

Proof of Theorem 1.2. This now follows from the main result (Theorem 1.1) in [St1, or rather from the proof of this theorem in section 5 in St1. What the latter assumes is a local non-integrability condition (LNIC), uniformly Lipschitz local stable holonomy maps and the so-called (see section 1 in St1]) regular distortion along unstable manifolds. In our case the flow is contact, so the condition (LNIC) 
follows from Proposition 6.2 in [St1]. What concerns regular distortion along unstable manifolds, one should note that section 5 in [St1 is only using a consequence of this property, namely the properties of cylinders described in Proposition 3.3 in [St1]. These properties are exactly the properties (a) and (b) in Theorem 4.2 above. Thus, under the assumptions of Theorem 1.2 above the argument from section 5 in [St1] applies and proves that the Ruelle transfer operators related to $f$ are eventually contracting for any Lipschitz real-valued function $f$ on $U$.

\section{REFERENCES}

[An] N. Anantharaman, Precise counting results for closed orbits of Anosov flows, Ann. Scient. Éc. Norm. Sup. 33 (2000), 33-56. MR1743718 (2002c:37048)

[B] R. Bowen, Symbolic dynamics for hyperbolic flows, Amer. J. Math. 95 (1973), 429-460. MR0339281 (49:4041)

[BP] L. Barreira and Ya. Pesin, Lyapunov exponents and smooth ergodic theory, University Lecture Series 23, American Mathematical Society, Providence, RI, 2002. MR.1862379 (2003a:37040)

[BR] R. Bowen and D. Ruelle, The ergodic theory of Axiom A flows, Invent. Math. 29 (1975), 181-202. MR0380889 (52:1786)

[CL] E. Coddington and N. Levinson, Theory of ordinary differential equations, McGraw-Hill, New York, 1955. MR0069338 (16:1022b)

[D] D. Dolgopyat, On decay of correlations in Anosov flows, Ann. of Math. (2) 147 (1998), 357-390. MR1626749 (99g:58073)

[Ha] B. Hasselblatt, Regularity of the Anosov splitting and of horospheric foliations, Ergod. Th. \& Dynam. Sys. 14 (1994), 645-666. MR 1304137 (95j:58130)

[HPS] M. Hirsch, C. Pugh and M. Shub, Invariant manifolds, Springer Lecture Notes in Mathematics, Vol. 583, 1977. MR0501173 (58:18595)

$[\mathrm{KH}]$ A. Katok and B. Hasselblatt, Introduction to the Modern Theory of Dynamical Systems, Cambridge Univ. Press, Cambridge, 1995. MR 1326374 (96c:58055)

[LY] F. Ledrappier and L.-S. Young, The metric entropy of diffeomorphisms: Part I: Characterization of measures satisfying Pesin's entropy formula, Ann. of Math. (2) 122 (1985), 509-539; Part II: Relations between entropy, exponents and dimension, Ann. of Math. (2) 122 (1985), 540-574. MR0819556 (87i:58101a) MR0819557 (87i:58101b)

[PP] W. Parry and M. Pollicott, Zeta functions and the periodic orbit structure of hyperbolic dynamics, Astérisque 187-188 (1990). MR1085356 (92f:58141)

[P1] Ya. Pesin, Characteristic exponents and smooth ergodic theory, Russian Mathematical Surveys 32 (1977), 55-114.

[P2] Ya. Pesin, Lectures on partial hyperbolicity and stable ergodicity, European Mathematical Society, Zürich, 2004. MR2068774 (2005j:37039)

[PeS1] V. Petkov and L. Stoyanov, Correlations for pairs of closed trajectories for open billiards, Nonlinearity 22 (2009), 2657-2679. MR2550690 (2011a:37080)

[PeS2] V. Petkov and L. Stoyanov, Analytic continuation of the resolvent of the Laplacian and the dynamical zeta function, Analysis and PDE 3 (2010), 427-489. MR2718260(2012a:35220)

[PeS3] V. Petkov and L. Stoyanov, Distribution of periods of closed trajectories in exponentially shrinking intervals, Comm. Math. Phys. 310 (2012), 675-704.

[PoS1] M. Pollicott and R. Sharp, Exponential error terms for growth functions of negatively curved surfaces, Amer. J. Math. 120 (1998), 1019-1042. MR.1646052 (99h:58148)

[PoS2] M. Pollicott and R. Sharp, Asymptotic expansions for closed orbits in homology classes, Geom. Dedicata 87 (2001), 123-160. MR1866845 (2003b:37051)

[PoS3] M. Pollicott and R. Sharp, Correlations for pairs of closed geodesics, Invent. Math. 163 (2006), 1-24. MR2208416 (2007a:37036)

[PS] C. Pugh and M. Shub, Ergodic attractors, Trans. Amer. Math. Soc. 312 (1989), 1-54. MR 983869 (90h:58057)

[PSW] C. Pugh, M. Shub and A. Wilkinson, Hölder foliations, Duke Math. J. 86 (1997), 517-546; Correction: Duke Math. J. 105 (2000), 105-106. MR1432307 (97m:58155) MR.1788044 (2001h:37057) 
[Ra] M. Ratner, Markov partitions for Anosov flows on $n$-dimensional manifolds, Israel J. Math. 15 (1973), 92-114. MR0339282 (49:4042)

[St1] L. Stoyanov, Spectra of Ruelle transfer operators for Axiom A flows, Nonlinearity 24 (2011), 1089-1120. MR2776112

[St2] L. Stoyanov, Non-integrability of open billiard flows and Dolgopyat type estimates, Ergod. Th. \& Dynam. Sys. 32 (2012), 295-313.

[St3] L. Stoyanov, Pinching conditions, linearization and regularity of Axiom A flows, preprint, 2010 (arXiv: math.DS:1010.1594)

School of Mathematics, University of Western Australia, Crawley, WA 6009, AusTRALIA

E-mail address: luchezar.stoyanov@uwa.edu.au 